\title{
THE SOFT TORUS AND APPLICATIONS TO ALMOST COMMUTING MATRICES
}

\author{
RUY EXEL
}

\begin{abstract}
The "Soft Torus" $A_{\varepsilon}$ is defined to be the universal $C^{*}$-algebra generated by a pair of unitaries for which the commutator has norm less than or equal to $\varepsilon$. We show that the $K$-theory of $A_{\varepsilon}$ is naturally isomorphic to the $K$-theory of the algebra of continuous functions on the two-torus although these algebras are not homotopically equivalent. This result is applied to give a new proof of the equality of certain invariants associated to almost commuting unitary matrices.
\end{abstract}

1. Introduction. The $C^{*}$-algebra $C\left(\mathbf{T}^{2}\right)$ of all continuous complex valued functions on the two-torus is well known to be the universal $C^{*}$-algebra generated by two commuting unitary elements.

Softening the commuting condition we define for all $\varepsilon$ in the real interval $[0,2]$ the "Soft Torus" $A_{\varepsilon}$ to be the universal $C^{*}$-algebra generated by a pair of elements $u_{\varepsilon}$ and $v_{\varepsilon}$, subject to the relations $u_{\varepsilon}^{*} u_{\varepsilon}=u_{\varepsilon} u_{\varepsilon}^{*}=1=v_{\varepsilon}^{*} v_{\varepsilon}=v_{\varepsilon} v_{\varepsilon}^{*}$ and $\left\|u_{\varepsilon} v_{\varepsilon}-v_{\varepsilon} u_{\varepsilon}\right\| \leq \varepsilon$.

Clearly if $\varepsilon$ is taken to be zero then $A_{\varepsilon}$ is nothing but an isomorphic model of the "hard torus" $C\left(\mathbf{T}^{2}\right)$. On the opposite extreme if $\varepsilon=2$ then $A_{\varepsilon}$ is the full $C^{*}$-algebra of the free group on two generators. The reader is referred to [1] for more information on the theory of $C^{*}$-algebras defined by generators and relations.

One of the main goals of this work is the computation of the $K$ theory groups of $A_{\varepsilon}$. It turns out that $A_{\varepsilon}$ has the same $K$-groups as $C\left(\mathbf{T}^{2}\right)$ when $\varepsilon<2$ (if $\varepsilon=2$ it is well known that $K_{0}\left(A_{\varepsilon}\right)=\mathbf{Z}$ and $\left.K_{1}\left(A_{\varepsilon}\right)=\mathbf{Z} \oplus \mathbf{Z}[4]\right)$. However we shall show that $A_{\varepsilon}$ is not in the homotopy class of $C\left(\mathbf{T}^{2}\right)$.

We say that two elements $u$ and $v$ in a $C^{*}$-algebra are $\varepsilon$-almost commuting if the commutation error $\|u v-v u\|$ is less than or equal to $\varepsilon$.

Several authors [3], [5], [8], [9], [10], [13], [17], [18] have investigated the properties of almost commuting complex matrices in what has been called, after Brown, Douglas and Fillmore's work on essentially normal operators [2], "quantitative BDF theory".

Recent works by Loring, Choi and myself [13], [3], [8] have introduced invariants which can detect obstructions to the existence of 
commuting approximations to given almost commuting pairs of unitary matrices.

Among these are the "winding invariant" and the " $K$-theory invariant" (defined below), denoted $\omega(u, v)$ and $\kappa(u, v)$ respectively, which are integers associated to pairs of unitary matrices $u$ and $v$ such that the commutation error is less than some constant $c$.

By its very definition $A_{\varepsilon}$ is the universal algebra for the almost commuting phenomenon as far as unitaries are concerned. That is, to every pair of $\varepsilon$-almost commuting unitaries in a $C^{*}$-algebra $A$ there is a unique homomorphism (always assumed to preserve the star operation) from $A_{\varepsilon}$ to $A$ such that the given unitaries are the images of $\dot{u}_{\varepsilon}$ and $v_{\varepsilon}$. Therefore, in a sense, studying $A_{\varepsilon}$ one studies simultaneously all almost commuting pairs of unitaries.

As we shall see the introduction of $A_{\varepsilon}$ provides a clearer understanding of the nature of the invariants $\kappa(u, v)$ and $\omega(u, v)$ mentioned above. The latter is related to the rotation number [6], [14] of a certain automorphism while $\kappa(u, v)$ is related to the trace on the $K_{0}$-group of the corresponding crossed product (as already indicated by Loring [13]).

Once we see things from this perspective we are able to give a new proof of the main result of [9] which states that $\kappa(u, v)=\omega(u, v)$. Our proof, unlike that of [9], falls entirely within the scope of $K$ theory and has the advantage of allowing the largest possible constant $c$ that is 2 (in the original definition of $\kappa(u, v)$ the constant is not explicit).

2. The soft torus. Let $z$ and $w$ denote the coordinate functions on the two-torus so that $z$ and $w$ represent two unitary elements in $C\left(\mathbf{T}^{2}\right)$. Since $z$ and $w$ commute, there is for every $\varepsilon$ in $[0,2]$ a unique homomorphism $\varphi_{\varepsilon}: A_{\varepsilon} \rightarrow C\left(\mathbf{T}^{2}\right)$ such that $\varphi_{\varepsilon}\left(u_{\varepsilon}\right)=z$ and $\varphi_{\varepsilon}\left(v_{\varepsilon}\right)=w$.

Our goal in this section is to prove that $\varphi_{\varepsilon}$ induces isomorphisms at the level of $K$-groups as long as $\varepsilon<2$.

2.1. LemMA. Let $u$ and $v$ be unitary elements in a $C^{*}$-algebra $A$ with $\|u-v\|=\varepsilon$ for some $\varepsilon<2$. Then there exists a continuous path (by a path we shall always mean a continuous function on the unit interval ) $u(t)$ of unitaries in $A$ such that $u(0)=u, u(1)=v$ and $\|u(t)-u(s)\| \leq \varepsilon$ for all $t$ and $s$ in $[0,1]$.

Proof. Note that $\left\|1-u^{-1} v\right\|=\varepsilon<2$ so that -1 is not in the spectrum of $u^{-1} v$. Let log denote the principal branch of the logarithm 
and put $h=\frac{1}{i} \log \left(u^{-1} v\right)$. One has $\|h\|=2 \arcsin \left(\frac{\varepsilon}{2}\right)$.

Let $u(t)=u e^{i t h}$ so that

$$
\|u(t)-u(s)\|=\left\|1-e^{i(s-t) h}\right\|=2 \sin \left(\frac{\|(s-t) h\|}{2}\right) \leq 2 \sin \left(\frac{\|h\|}{2}\right)=\varepsilon
$$

so that $u(t)$ is the desired path.

In order to compute the $K$-theory of $\varphi_{\varepsilon}$ we shall first show that $A_{\varepsilon}$ is isomorphic to a crossed product $B_{\varepsilon} \times_{\alpha_{\varepsilon}} \mathbf{Z}$. Here $B_{\varepsilon}$ is defined to be the universal $C^{*}$-algebra generated by a countable set $\left\{u_{n}: n \in \mathbf{Z}\right\}$ subject to the conditions that each $u_{n}$ is unitary and that $\| u_{n+1}-$ $u_{n} \| \leq \varepsilon$ for all $n$.

If $z$ denotes the canonical generator of the algebra $C\left(\mathbf{S}^{1}\right)$ of continuous functions on the unit circle, we let $\psi_{\varepsilon}: B_{\varepsilon} \rightarrow C\left(\mathbf{S}^{1}\right)$ be the unique homomorphism such that $\psi_{\varepsilon}\left(u_{n}\right)=z$ for all $n$.

2.2. THEOREM. If $\varepsilon<2$ then $\psi_{\varepsilon}$ is a homotopy equivalence between $B_{\varepsilon}$ and $C\left(\mathbf{S}^{1}\right)$.

Proof. Let $\sigma: C\left(\mathbf{S}^{1}\right) \rightarrow B_{\varepsilon}$ be given by $\sigma(z)=u_{0}$. Clearly $\psi_{\varepsilon} \sigma$ is the identity map of $C\left(\mathbf{S}^{1}\right)$. We now prove that $\sigma \psi_{\varepsilon}$ is homotopic to the identity of $B_{\varepsilon}$.

For any integer $p \geq 0$ let $u_{p}^{+}(t)$ and $u_{p}^{-}(t)$ be continuous paths of unitaries in $B_{\varepsilon}$ such that

$$
u_{p}^{ \pm}(0)=u_{ \pm p}, \quad u_{p}^{ \pm}(1)=u_{ \pm(p+1)}
$$

satisfying the conditions of Lemma (2.1). In particular we have $\left\|u_{p}^{ \pm}(t)-u_{ \pm p}\right\| \leq \varepsilon$ for all $t$.

Concatenating these paths and performing a reparametrization we obtain paths $\gamma^{+}(t)$ and $\gamma^{-}(t)$, defined for $0 \leq t<1$, such that for all $p \geq 0$ one has $\gamma^{ \pm}\left(\frac{p}{p+1}\right)=u_{ \pm p}$. Moreover we may require that

$$
\frac{p}{p+1} \leq t \leq \frac{p+1}{p+2} \Rightarrow\left\|\gamma^{ \pm}(t)-u_{ \pm p}\right\| \leq \varepsilon
$$

For each integer $n$ define a path $v_{n}$ in $B_{\varepsilon}$ by

$$
v_{n}(t)= \begin{cases}\gamma^{\operatorname{sgn}(n)}(t) & \text { if } 0 \leq t<\frac{|n|}{|n|+b} \\ u_{n} & \text { if } \frac{|n|}{|n|+1} \leq t \leq 1\end{cases}
$$

Here $\operatorname{sgn}(n)$ should be taken to be " + " or " - " according to the sign of $n$. Note that regardless of the choice made for $\operatorname{sgn}(0)$ one has that $v_{0}(t)$ is constantly equal to $u_{0}$. 
Observe that for all $t$ and $n$ one has that

$$
\left\|v_{n+1}(t)-v_{n}(t)\right\| \leq \varepsilon .
$$

Therefore for all $t$ in $[0,1]$ we let $H_{t}$ be the unique endomorphism of $B_{\varepsilon}$ such that $H_{t}\left(u_{n}\right)=v_{n}(t)$. Clearly this yields a homotopy from $\sigma \psi_{\varepsilon}$ to the identity map of $B_{\varepsilon}$.

A pictorical description of our argument is this: think of a long road, in the middle of which there is a post-office. Two postmen leave the post-office in opposite directions and when the clock shows $\frac{n}{n+1}$ each postman delivers the $n$th letter to the $n$th house (the 0th letter was delivered to the post-office itself). Although the postmen will have to reach an infinite speed to achieve such a difficult task, each individual letter will have been safely delivered by then. No letter will ever lose sight of the next because either they are both in the post-bag or one has been delivered while the postman travels a short distance to the next house or they both finally lie in neighbouring post-boxes. This concludes the proof.

Let $\alpha_{\varepsilon}$ be the automorphism of $B_{\varepsilon}$ specified by $\alpha_{\varepsilon}\left(u_{n}\right)=u_{n+1}$.

2.3. Proposition. For all $\varepsilon \in[0,2]$ one has that $A_{\varepsilon}$ is isomorphic to the crossed product $B_{\varepsilon} \times_{\alpha_{\varepsilon}} \mathbf{Z}$.

Proof. Let $y$ be the canonical implementing unitary of $B_{\varepsilon} \times{ }_{\alpha_{\varepsilon}} \mathrm{Z}$ so that $y u_{n} y^{-1}=u_{n+1}$. Then $\left\|u_{0} y-y u_{0}\right\|=\varepsilon$. Therefore there exists a unique homomorphism $\varphi: A_{\varepsilon} \rightarrow B_{\varepsilon} \times_{\alpha_{\varepsilon}} \mathbf{Z}$ such that $\varphi\left(u_{\varepsilon}\right)=u_{0}$ and $\varphi\left(v_{\varepsilon}\right)=y$.

On the other hand note that $\left\|v_{\varepsilon}^{n+1} u_{\varepsilon} v_{\varepsilon}^{-(n+1)}-v_{\varepsilon}^{n} u_{\varepsilon} v_{\varepsilon}^{-n}\right\| \leq \varepsilon$. So there is a homomorphism $\psi: B_{\varepsilon} \rightarrow A_{\varepsilon}$ with $\psi\left(u_{n}\right)=v_{\varepsilon}^{n} u_{\varepsilon} v_{\varepsilon}^{-n}$. Since $v_{\varepsilon} \psi\left(u_{n}\right) v_{\varepsilon}^{-1}=\psi\left(u_{n+1}\right)$ one can extend $\psi$ to $B_{\varepsilon} \times_{\alpha_{\varepsilon}} \mathbf{Z}$ such that $\psi(y)=v_{\varepsilon}$. Clearly $\varphi$ and $\psi$ are each others inverse.

Recall that $\varphi_{\varepsilon}: A_{\varepsilon} \rightarrow C\left(\mathbf{T}^{2}\right)$ is defined by $\varphi_{\varepsilon}\left(u_{\varepsilon}\right)=z$ and $\varphi_{\varepsilon}\left(v_{\varepsilon}\right)=$ $w$.

2.4. THEOREM. If $\varepsilon<2$ then

$$
\varphi_{\varepsilon_{*}}: K_{i}\left(A_{\varepsilon}\right) \rightarrow K_{i}\left(C\left(\mathbf{T}^{2}\right)\right)
$$

is an isomorphism for $i=1,2$.

Proof. Regard $C\left(\mathbf{T}^{2}\right)$ as $C\left(\mathbf{S}^{1}\right) \times{ }_{\text {id }} \mathbf{Z}$ where the copy of $C\left(\mathbf{S}^{1}\right)$ is generated by $z$, the generator of $\mathbf{Z}$ is identified with $w$ and the action considered is the trivial action. 
Note that $\psi_{\varepsilon}$ is a covariant map which therefore extends to a homomorphism between the corresponding crossed product algebras $B_{\varepsilon} \times_{\alpha_{\varepsilon}} \mathbf{Z}$ and $C\left(\mathbf{S}^{1}\right) \times_{\mathrm{id}} \mathbf{Z}$.

Identifying $A_{\varepsilon}$ with $B_{\varepsilon} \times_{\alpha_{\varepsilon}} \mathbf{Z}$ according to Proposition (2.3) this extended map is clearly the same as $\varphi_{\varepsilon}$.

Note that in the Pimsner-Voiculescu exact sequence [15] for these crossed products the maps usually denoted " $1-\alpha_{*}^{-1}$ " both vanish. The naturality of the Pimsner-Voiculescu maps thus provides the following commutative diagram with exact rows.

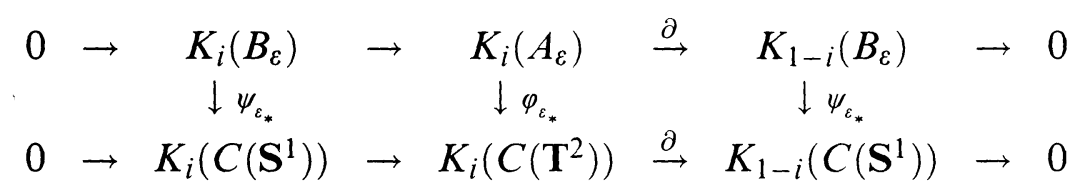

An application of the five lemma thus completes the proof.

2.5. Corollary. If $\varepsilon<2$ then $K_{0}\left(A_{\varepsilon}\right)=\mathbf{Z} \oplus \mathbf{Z}$ and $K_{1}\left(A_{\varepsilon}\right)=$ $\mathbf{Z} \oplus \mathbf{Z}$.

The Bott element, as it is sometimes called, is the element $b \in$ $K_{0}\left(C\left(\mathbf{T}^{2}\right)\right)$ characterized (up to a sign convention) by the fact that $b$ generates the kernel of $\tau_{*}: K_{0}\left(C\left(\mathbf{T}^{2}\right)\right) \rightarrow \mathbf{Z}$ where $\tau_{*}$ is the map induced by any unital trace $\tau$ on $C\left(\mathbf{T}^{2}\right)$.

Another characterization of $b$ (up to the same sign convention) is that it generates the second spherical homology group $\mathrm{H}_{2}\left(\mathrm{C}\left(\mathbf{T}^{2}\right)\right)$ (see [7]). The image of $b$ under the connecting map $\partial: K_{0}\left(C\left(\mathbf{T}^{2}\right)\right) \rightarrow$ $K_{1}\left(C\left(\mathbf{S}^{1}\right)\right)$ is a generator of the latter group. We choose our sign convention so that $\partial(b)$ equals the $K_{1}$-class of $z^{-1}$.

Denote by $b_{\varepsilon}$ the element in $K_{0}\left(A_{\varepsilon}\right)$ defined by $b_{\varepsilon}=\varphi_{\varepsilon_{*}}^{-1}(b)$. The reader should however be warned that $b_{\varepsilon}$ doesn't share the same properties of $b$. In fact we have the following

2.6. TheOREM. If $\varepsilon<2$ then the unital traces of $A_{\varepsilon}$, once dropped to $K_{0}$, form a separating family of maps.

We shall pospone the proof of this result to the next section. Nevertheless the following consequences should be noted.

2.7. COROLlaRY. If $\varepsilon<2$ then the second spherical homology group $\mathrm{H}_{2}\left(A_{\varepsilon}\right)$ is zero. Therefore $A_{\varepsilon}$ is not homotopically equivalent to $C\left(\mathbf{T}^{2}\right)$. 
Proof. Traces vanish on elements of the second spherical filtration subgroup $F_{2} K_{0}\left(A_{\varepsilon}\right)$ [7] which are representatives of elements in $H_{2}$. Therefore the conclusion follows from Theorem (2.6).

A last important remark should perhaps be made before concluding this section. That is, elements in $K K(A, B)$, where $A$ and $B$ are $C^{*}$-algebras, are known to induce homomorphisms at the level of $K$ groups [12]. Nevertheless these maps are not required to preserve the corresponding spherical filtrations. In other words the argument above does not rule out the existence of a $K K$-equivalence between the "soft" and the "hard" tori.

3. Invariants for almost commuting unitaries. Let $A$ be a $C^{*}$-algebra and $(u, v)$ be a pair of unitaries in $A$ such that $\|u v-v u\| \leq \varepsilon$ for some $\varepsilon<2$. As mentioned in the introduction there is a unique homomorphism $\rho: A_{\varepsilon} \rightarrow A$ such that $\rho\left(u_{\varepsilon}\right)=u$ and $\rho\left(v_{\varepsilon}\right)=v$.

The " $K$-theory invariant" of the pair $(u, v)$ is the element of $K_{0}(A)$ defined by

$$
\kappa(u, v)=\rho_{*}\left(b_{\varepsilon}\right) .
$$

This invariant was introduced by Loring [13] for small values of $\varepsilon$ in order to give a $K$-theoretical proof of a former result of Voiculescu [17] asserting that some pairs of almost commuting unitaries cannot be approximated by commuting pairs (see also [3]). Observe that $\kappa(u, v)$ does not depend on $\varepsilon$ as long as $\varepsilon \geq\|u v-v u\|$.

In the case where $A$ is the algebra of complex $n \times n$ matrices then $K_{0}(A) \cong \mathbf{Z}$ so we may think of $\kappa(u, v)$ as an integer identifying it with its image under $\operatorname{Tr}_{*}$ (here as in the sequel $\operatorname{Tr}$ denotes the standard trace on the algebra of complex matrices).

Still assuming that $A=M_{n}(\mathbf{C})$ we define the "winding invariant" of the pair $(u, v)$, denoted $\omega(u, v)$, to be the winding number of the closed complex path

$$
\gamma(t)=\operatorname{det}(t v u+(1-t) u v)
$$

around zero. That $\gamma$ is never zero follows from $\|u v-v u\|<2$.

The main theorem of [9] asserts that $\kappa(u, v)=\omega(u, v)$ as long as $\|u v-v u\|<c$ for some small constant $c$ (which according to the main rule of this game is independent of $n$ ). The purpose of the present section is to give a new proof of this fact which has two advantages. First it is a purely $K$-theoretical proof. Second it works for the largest possible value of $c$ that is 2 . 
3.1. Lemma. Let $u$ and $v$ be unitary $n \times n$ complex matrices with $\|u v-v u\|=\varepsilon$ for some $\varepsilon<2$. Then

$$
\omega(u, v)=\frac{1}{2 \pi i} \operatorname{Tr}\left(\log \left(v u v^{-1} u^{-1}\right)\right),
$$

where $\log$ denotes the principal branch of the logarithm.

Proof. First note that the right-hand side is well defined since $\| 1$ $v u v^{-1} u^{-1} \|<2$ implies that -1 is not in the spectrum of $v u v^{-1} u^{-1}$. Therefore let $h=\frac{1}{i} \log \left(v u v^{-1} u^{-1}\right)$ and note that $\|h\|=2 \arcsin \left(\frac{\varepsilon}{2}\right)$.

We claim that the paths

$$
t \mapsto e^{i t h} u v \quad \text { and } t \mapsto t v u+(1-t) u v
$$

are homotopic (end points fixed) in $\mathrm{GL}_{n}(\mathbf{C})$. In fact the linear path (in the space of curves in $\mathrm{GL}_{n}(\mathbf{C})$ ) provides such a homotopy. To see this it is enough to check that

$$
\left\|t v u+(1-t) u v-e^{i t h} u v\right\|<1
$$

for all $t$ since $e^{i t h} u v$ is unitary. But

$$
\left\|t v u+(1-t) u v-e^{i t h} u v\right\|=\left\|t e^{i h}+(1-t)-e^{i t h}\right\|
$$

which can be proven by the spectral theorem and elementary trigonometry to be less than or equal to $1-\sqrt{1-\left(\frac{\varepsilon}{2}\right)^{2}}$.

Thus $\omega(u, v)$ equals the winding number of

$$
t \mapsto \operatorname{det}\left(e^{i t h} u v\right)
$$

which can be easily computed and equals $\frac{1}{2 \pi} \operatorname{Tr}(h)$.

The major tool we shall need in the following is a theorem on rotation numbers for automorphisms of $C^{*}$-algebras [6], [14]. A brief description of this result is in order.

Let $\alpha$ be an automorphism of a unital $C^{*}$-algebra $A$ and let $\tau$ be an $\alpha$-invariant trace on $A$ such that the pair $(A, \tau)$ is an integral algebra (in the sense that the range of $\tau_{*}$ on $K_{0}(A)$ is contained in Z).

Denote by $\partial: K_{0}\left(A \times_{\alpha} \mathbf{Z}\right) \rightarrow K_{1}(A)$ the connecting map of the Pimsner-Voiculescu sequence [15].

Theorem (V.12) of [6] asserts that for every $x$ in $K_{0}\left(A \times_{\alpha} Z\right)$ one has

$$
\exp \left(2 \pi i \tau_{*}(x)\right)=\operatorname{det}_{\tau}\left(\alpha\left(u^{-1}\right) u\right)
$$

where $u$ is any unitary matrix over $A$ whose $K_{1}$-class is $\partial(x)$. 
Here the occurrence of $\tau$ in the left-hand side refers to the canonical extension of $\tau$ to $A \times{ }_{\alpha} \mathbf{Z}$, while $\operatorname{det}_{\tau}$ is a determinant associated to $\tau[6],[11]$.

With this at hand we may now prove the main theorem of this section.

3.2. TheOREM. Let $u$ and $v$ be unitary $n \times n$ complex matrices with $\|u v-v u\|=\varepsilon$ for some $\varepsilon<2$. Then $\kappa(u, v)=\omega(u, v)$.

Proof. Denote by $\rho: A_{\varepsilon} \rightarrow M_{n}(\mathbf{C})$ the unique homomorphism such that $\rho\left(u_{\varepsilon}\right)=u$ and $\rho\left(v_{\varepsilon}\right)=v$. Define a unital trace on $A_{\varepsilon}$ by $\tau=\frac{1}{n} \operatorname{Tr} \circ \rho$.

Identify $B_{\varepsilon}$ as a subalgebra of $A_{\varepsilon}$ under the isomorphism of Proposition (2.3). So $\tau$ gives by restriction an $\alpha_{\varepsilon}$-invariant trace on $B_{\varepsilon}$.

Note that $\tau$ is an integral trace on $B_{\varepsilon}$ although $\frac{1}{n} \operatorname{Tr}$ is not an integral trace on $M_{n}(\mathbf{C})$, the reason being that on the homotopy class of $C\left(\mathbf{S}^{1}\right)$ any unital trace is integral!

Whenever a trace on a $C^{*}$-algebra is invariant under a given automorphism it is possible to extend it in a canonical way to the crossed product of the algebra by $\mathbf{Z}$. According to this let $\tilde{\tau}$ be the canonical extension of $\left.\tau\right|_{B_{\varepsilon}}$ to $A_{\varepsilon}$ but beware that $\tilde{\tau}$ is often different from $\tau$ on $A_{\varepsilon}$. Nevertheless $\tau_{*}=\tilde{\tau}_{*}$ on $K_{0}\left(A_{\varepsilon}\right)$ as shown below.

3.3. Lemma. Let $\alpha$ be an automorphism of a $C^{*}$-algebra $A$ and let $\tau_{1}$ and $\tau_{2}$ be traces on $A \times_{\alpha} \mathbf{Z}$ such that $\tau_{1}=\tau_{2}$ on $A$. Then $\tau_{1_{*}}=\tau_{2_{*}}$ on $K_{0}\left(A \times \times_{\alpha} \mathbf{Z}\right)$.

Proof. Without loss of generality we may assume $A$ unital. Let $p$ be a projection (selfadjoint idempotent) in $M_{k}\left(A \times_{\alpha} \mathbf{Z}\right)$ for some $k$. It suffices to prove that $\tau_{1}(p)=\tau_{2}(p)$.

Again without loss of generality we assume that $k=1$. Let $\hat{\alpha}$ be the dual action of $\mathbf{S}^{1}$ on $A \times{ }_{\alpha} \mathbf{Z}$. For $i=1,2$ observe that $\tau_{i}\left(\hat{\alpha}_{z}(p)\right)$ does not depend on $z \in \mathbf{S}^{1}$ since close projections are equivalent. Thus

$$
\tau_{i}(p)=\int_{\mathbf{S}^{1}} \tau_{i}\left(\hat{\alpha}_{z}(p)\right) d z=\tau_{i}(E(p))
$$

where $E$ is the canonical conditional expectation [16] from $A \times{ }_{\alpha} \mathbf{Z}$. to $A$. Hence $\tau_{1}(p)=\tau_{2}(p)$.

Returning to the proof of Theorem (3.2) we have

$$
\tilde{\tau}_{*}\left(b_{\varepsilon}\right)=\tau_{*}\left(b_{\varepsilon}\right)=\frac{1}{n} \operatorname{Tr}_{*} \rho_{*}\left(b_{\varepsilon}\right)=\frac{1}{n} \kappa(u, v) .
$$


Next observe that the class of $u_{0}^{-1}$ in $K_{1}\left(B_{\varepsilon}\right)$ is $\partial\left(b_{\varepsilon}\right)$, a fact which follows from the sign convention we made when introducing the Bott element and the naturality of the Pimsner-Voiculescu connecting maps. So if we apply Theorem (V.12) of [6] we obtain

$$
\exp \left(\frac{2 \pi i}{n} \kappa(u, v)\right)=\operatorname{det}_{\tau}\left(\alpha_{\varepsilon}\left(u_{0}\right) u_{0}^{-1}\right) .
$$

On the other hand we have

$$
\alpha_{\varepsilon}\left(u_{0}\right) u_{0}^{-1}=u_{1} u_{0}^{-1}=\exp \left(\log \left(u_{1} u_{0}^{-1}\right)\right)
$$

so

$$
\operatorname{det}\left(\alpha_{\varepsilon}\left(u_{0}\right) u_{0}^{-1}\right)=\exp \left(\tau\left(\log \left(u_{1} u_{0}^{-1}\right)\right)\right)
$$

But

$$
\begin{aligned}
\tau\left(\log \left(u_{1} u_{0}^{-1}\right)\right) & =\frac{1}{n} \operatorname{Tr}\left(\rho\left(\log \left(u_{1} u_{0}^{-1}\right)\right)\right)=\frac{1}{n} \operatorname{Tr}\left(\log \left(\rho\left(u_{1} u_{0}^{-1}\right)\right)\right) \\
& =\frac{1}{n} \operatorname{Tr}\left(\log \left(v u v^{-1} u^{-1}\right)\right)=\frac{2 \pi i}{n} \omega(u, v) .
\end{aligned}
$$

Therefore

$$
\exp \left(\frac{2 \pi i}{n} \kappa(u, v)\right)=\exp \left(\frac{2 \pi i}{n} \omega(u, v)\right)
$$

so that $\frac{\kappa(u, v)-\omega(u, v)}{n} \in \mathbf{Z}$.

To conclude the proof observe that upon replacing $u$ by $u \oplus I_{m}$ and $v$ by $v \oplus I_{m}$ where $I_{m}$ is the $m \times m$ identity matrix, neither $\kappa(u, v)$ nor $\omega(u, v)$ will change. Thus we conclude that $\frac{\kappa(u, v)-\omega(u, v)}{n+m} \in \mathbf{Z}$ for all $m$. That is $\kappa(u, v)=\omega(u, v)$.

The success in exploiting the $K$-theory of the soft torus may lead one to believe that something along these lines could be tried for the "soft" algebra related to almost commuting selfadjoint matrices, the first and perhaps the least understood of all "almost commuting problems".

We mean of course the universal $C^{*}$-algebra $C_{\varepsilon}$ generated by a pair of selfadjoint elements $h_{\varepsilon}$ and $k_{\varepsilon}$ subject to the conditions $\left\|h_{\varepsilon}\right\| \leq 1$, $\left\|k_{\varepsilon}\right\| \leq 1$ and $\left\|h_{\varepsilon} k_{\varepsilon}-k_{\varepsilon} h_{\varepsilon}\right\| \leq \varepsilon$.

However $C_{\varepsilon}$ is a contractible $C^{*}$-algebra as it is easily seen by considering the path $f_{t}$ of endomorphisms of $C_{\varepsilon}$ given by $f_{t}\left(h_{\varepsilon}\right)=t h_{\varepsilon}$ and $f_{t}\left(k_{\varepsilon}\right)=t k_{\varepsilon}$.

To conclude let us give a proof of Theorem (2.6). 
Proof of Theorem 2.6. Recall that Voiculescu's unitaries [17] (see also [8]) $S_{n}$ and $\Omega_{n}$ are defined for all $n \geq 2$ by

$$
S_{n}=\left(\begin{array}{ccccc}
0 & & & & 1 \\
1 & 0 & & & \\
& 1 & 0 & & \\
& & \ddots & & \\
& & & 1 & 0
\end{array}\right) \text { and } \Omega_{n}=\left(\begin{array}{ccccc}
\omega_{n} & & & & \\
& \omega_{n}^{2} & & & \\
& & \omega_{n}^{3} & & \\
& & & \ddots & \\
& & & & \omega_{n}^{n}
\end{array}\right) \text {, }
$$

where $\omega_{n}=e^{2 \pi i / n}$. It can be easily shown that $\left\|S_{n} \Omega_{n}-\Omega_{n} S_{n}\right\|$ tends to zero as $n$ tends to infinity.

Given $\varepsilon$ let $n_{0}$ be such that $\left\|S_{n} \Omega_{n}-\Omega_{n} S_{n}\right\| \leq \varepsilon$ whenever $n \geq n_{0}$. For each $n \geq n_{0}$ let $\rho_{n}: A_{\varepsilon} \rightarrow M_{n}(\mathbf{C})$ be defined by $\rho_{n}\left(u_{\varepsilon}\right)=S_{n}$ and $\rho_{n}\left(v_{\varepsilon}\right)=\Omega_{n}$. Let $\tau_{n}$ be the unital trace on $A_{\varepsilon}$ given by $\tau_{n}=$ $\frac{1}{n} \operatorname{Tr} \circ \rho_{n}$. We claim that the set

$$
\left\{\tau_{n_{*}}: n \geq n_{0}\right\}
$$

is a separating family.

In fact denote by [1] $]_{0}$ the $K_{0}$-class of the unit and note that $K_{0}\left(A_{\varepsilon}\right)$ is then generated by $[1]_{0}$ and $b_{\varepsilon}$. We have that $\tau_{n_{*}}\left([1]_{0}\right)=1$ while

$$
\tau_{n}\left(b_{\varepsilon}\right)=\frac{1}{n} \kappa\left(S_{n}, \Omega_{n}\right)=\frac{1}{n} \omega\left(S_{n}, \Omega_{n}\right)=\frac{1}{n}
$$

where the last step follows by direct computation.

So for $r, s \in \mathbf{Z}$ we have

$$
\tau_{n_{*}}\left(r[1]_{0}+s b_{\varepsilon}\right)=r+\frac{s}{n}
$$

from which the conclusion follows.

\section{REFERENCES}

[1] B. Blackadar, Shape theory for $C^{*}$-algebras, Math. Scand. 56 (1985), 249-275.

[2] L. G. Brown, R. G. Douglas and P. A. Filmore, Extensions of $C^{*}$-algebras and K-homology, Ann. of Math., 105 (1977), 265-324.

[3] M. D. Choi, Almost commuting matrices need not be nearly commuting, Proc. Amer. Math. Soc., 102 (1988), 529-533.

[4] J. Cuntz, The K-groups for free products of $C^{*}$-algebras, Operator Algebras and Applications (ed. R. V. Kadison), Proc. Sympos. Pure Math., vol. 38, Amer; Math. Soc., Providence, RI (1982), 81-84.

[5] K. R. Davidson, Almost commuting hermitian matrices, Math. Scand., 56 (1985), 222-240.

[6] R. Exel, Rotation numbers for automorphisms of $C^{*}$-algebras, Pacific J. Math., 127 (1987), 31-89. 
[7] R. Exel and T. A. Loring, Extending cellular cohomology to $C^{*}$-algebras, Trans. Amer. Math. Soc. 329 (1992), 141-160.

[8] _ Almost commuting unitary matrices, Proc. Amer. Math. Soc., 106 (1989), 913-915.

[9] _ Invariants of almost commuting unitaries, J. Funct. Anal., 95 (1991), 364-376.

[10] P. R. Halmos, Some unsolved problems of unknown depth about operators on Hilbert space, Proc. Roy. Soc. Edinburgh Sect. A, 76 (1976), 67-76.

[11] P. de la Harpe and G. Skandalis, Déterminant associé à une trace sur une algèbre de Banach, Ann. Inst. Fourier, 34 (1984).

[12] G. G. Kasparov, The operator $K$-functor and extensions of $C^{*}$-algebras, Izv. Akad. Nauk SSSR, Ser. Mat., 44 (1980), 571-636.

[13] T. A. Loring, $K$-theory and asymptotically commuting matrices, Canad. J. Math., 40 (1988), 197-216.

[14] M. Pimsner, Range of traces on $K_{0}$ of reduced crossed products by free groups, in Operator algebras and their connections with topology and ergodic theory, Proceedings, Bustenni, Romania 1983; Springer Lect. Notes in Math., 1132 (1985), 374-408.

[15] M. Pimsner and D. Voiculescu, Exact sequences for $K$ groups and Ext groups of certain crossed product $C^{*}$-algebras, J. Operator Theory 4 (1980), 93-118.

[16] M. A. Rieffel, Induced representations of $C^{*}$-algebras, Adv. Math., 13 (1974), 176-257.

[17] D. Voiculescu, Asymptotically commuting finite rank unitary operators without commuting approximants, Acta Sci. Math. (Szeged), 45 (1983), 429-431.

[18] _ Remarks on the singular extension in the $C^{*}$-algebra of the Heisenberg group, J. Operator Theory, 5 (1981), 147-170.

Received September 16, 1991. Partially supported by CNPq, Brazil.

Departamento de Matemática

Universidade de São Paulo

Caixa Postal 20570

01498 São Paulo SP, Brasil 
